\title{
Kinematically complete measurements of strong field ionisation with mid-IR pulses
}

\author{
Michael G. Pullen ${ }^{1}$, Judith Durá ${ }^{1 *}$, Benjamin Wolter $^{1}$, \\ Matthias Baudisch ${ }^{1}$, Michaël Hemmer ${ }^{1}$, Nicolas Camus ${ }^{2}$, Arne \\ Senftleben $^{3}$, Claus Dieter Schroeter ${ }^{2}$, Robert Moshammer ${ }^{2}$, \\ Joachim Ullrich ${ }^{2,4}$ \& Jens Biegert ${ }^{1,5}$ \\ ${ }^{1}$ ICFO-Institut de Ciències Fotòniques, Av. Carl Friedrich Gauss 3, 08860 \\ Castelldefels (Barcelona), Spain \\ ${ }^{2}$ Max-Planck-Institut für Kernphysik, Saupfercheckweg 1, 69117 Heidelberg, \\ Germany \\ ${ }^{3}$ Institut für Physik und CINSaT, Universität Kassel, Heinrich-Plett-Strasse 40, \\ 34132 Kassel, Germany \\ ${ }^{4}$ Physikalisch-Technische Bundesanstalt (PTB), Bundesallee 100, 38116 \\ Braunschweig, Germany \\ ${ }^{5}$ ICREA-Institució Catalana de Recerca i Estudis Avançats, 08010 Barcelona, Spain \\ *Present address: Max-Born Institute (MBI), Max-Born Strasse 2A, 12489 Berlin, \\ Germany \\ E-mail: michael.pullen@icfo.eu
}

\begin{abstract}
Recent observations of three unique peaks near $1 \mathrm{eV}, 100 \mathrm{meV}$ and $1 \mathrm{meV}$ in the electron spectra generated by ionisation using intense mid-IR pulses have challenged the current understanding of strong field ionisation. The results came as a surprise as they could not be reproduced by the standard version of the commonly used strong field approximation. We present results showing the simultaneous measurement of all three low energy ranges at high resolution. This capability is possible due to a unique experimental combination of a high repetition rate mid-IR source, which allows probing deep in the quasi-static regime at high data rates, with a reaction microscope, which allows high resolution three dimensional imaging of the electron momentum distribution.
\end{abstract}




\section{Strong field ionisation}

Strong-field (SF) ionisation is the fundamental physical process behind highly nonlinear phenomena such as multi-photon ionisation, above threshold ionisation, sequential and non-sequential double ionisation, and high harmonic generation. These phenomena are used extensively in modern research topics such as tomographic imaging [1], laser induced electron diffraction [2] and attosecond science [3]. Extraction of physical parameters from the associated experimental observations requires a comparison to theoretical simulations. Fundamental understanding of the SF ionisation process is therefore of paramount importance to the continual progression of these research fields. It is desirable then for theoretical models to qualitatively reproduce the observed features in the electron and ion ionisation spectra.

Current understanding of SF ionisation is based upon the seminal work of Keldysh from 1964 where ionisation induced by an intense electromagnetic wave of low frequency compared to the ionisation potential was considered [4]. In this work it was noted that there are two regimes in which ionisation can occur: the so called multi-photon (MP) ionisation regime, which can be understood by perturbation theory, and the commonly termed tunneling regime for which perturbation theory fails. In the tunneling regime the laser electric field can be considered quasi-static and the interaction approaches the classical limit. Henceforth this region will be called the quasi-static (QS) ionisation regime. The unitless Keldysh parameter $(\gamma)$, which was introduced in Ref. [4], provides an eloquent way to distinguish between the two ionisation pathways. In atomic units, which are used in this paper unless otherwise stated, this parameter is given by,

$$
\gamma=\sqrt{\frac{I_{P}}{2 U_{P}}},
$$

and is a function of the ionisation potential $\left(I_{P}\right)$ of the target and the cycle averaged ponderomotive energy of the electron in the laser field $\left(U_{P}\right)$, which in turn is dependent on the laser peak intensity $(I)$, wavelength $(\lambda)$ and frequency $(\omega)$,

$$
U_{P}=\frac{I \lambda^{2}}{16 \pi^{2}}=\frac{I}{4 \omega^{2}}
$$

The MP regime is encountered when $\gamma>>1$ while the QS regime is a more appropriate description when $\gamma<<1$. The two regimes are presented in Fig. 1a for a range of currently achievable laser peak intensities and wavelengths. The presented wavelengths correspond to the ubiquitous Ti:Sapphire technology $(0.8 \mu \mathrm{m})$, possible optical parametric amplification (OPA) outputs $(1.3 \mu \mathrm{m}$ and $2.0 \mu \mathrm{m})$ and the output of the optical parametric chirped pulse amplification (OPCPA) system presented in this work $(3.1 \mu \mathrm{m})$ [5]. The transition from MP ionisation (blue shading) to QS ionisation (red shading) is gradual and a region exists near $\gamma \sim 1$ where both regimes contribute (white). Most experiments using a wavelength of $0.8 \mu \mathrm{m}$ operate within this mixed regime. 


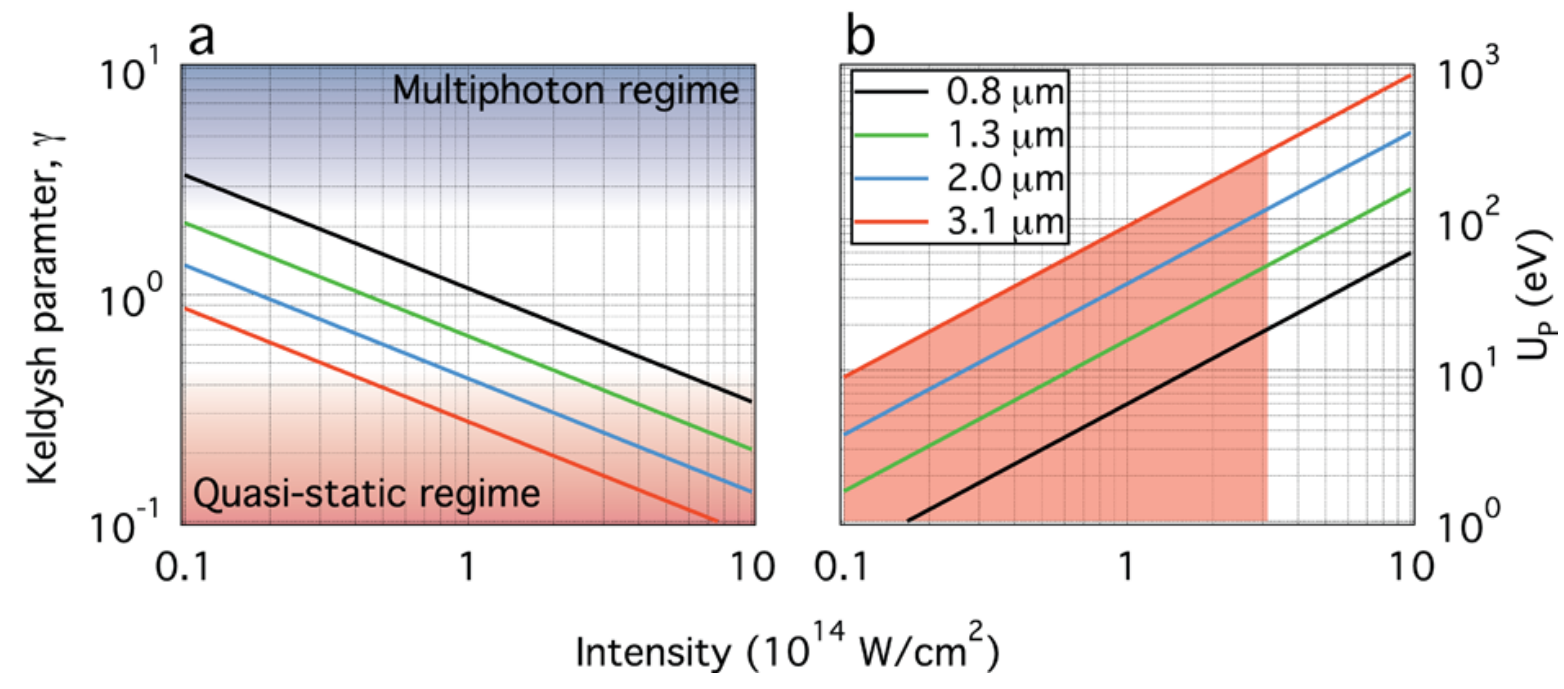

Figure 1. The location of the different ionisation regimes (a) and the maximum achievable ponderomotive energies $U_{P}$ (b) as a function of the peak laser intensity for different laser wavelengths. The blue shaded region in (a) represents the perturbative MP regime while the red shaded region represents the non-perturbative QS limit. The red shaded region in (b) indicates the range over which the laser used in this work operates.

The benefits of scaling to longer wavelengths are evident in Fig. 1. Increasing the laser wavelength results in an increased ability to probe deeper in the QS regime. To reach a Keldysh parameter of $\gamma=0.4$, for example, peak intensities of $\sim 4.3 \times 10^{14} \mathrm{~W} / \mathrm{cm}^{2}$ and $\sim 0.3 \times 10^{14} \mathrm{~W} / \mathrm{cm}^{2}$ are required for wavelengths of $0.8 \mu \mathrm{m}$ and $3.1 \mu \mathrm{m}$, respectively. At these intensities, and for a typical pulse duration of $\sim 50 \mathrm{fs}$, Perelomov-Popov-Terent'ev (PPT) calculations [6] predict that the ground state of Ar would be depleted for $0.8 \mu \mathrm{m}$ but unity for $3100 \mu \mathrm{m}$. The ability to probe deeper into the QS regime by purely increasing the laser peak intensity of short wavelength radiation is therefore limited.

Figure $1 \mathrm{~b}$ presents the corresponding increase in achievable electron energies for the same wavelengths used in Fig. 1a. With long wavelength sources, ponderomotive energies of hundreds of eV can be obtained. Such electrons can be utilised in laserinduced electron diffraction [2] experiments and to generate soft X-rays via high harmonic generation [7]. The shaded red region indicates the range of ponderomotive energies that the OPCPA system in this paper can generate. To be able to perform both low $(1 \mathrm{meV})$ and high (hundreds of eV) electron energy experiments an electron detection system with a five orders of magnitude dynamic range, such as the reaction microscope (ReMi) used here, is required.

\subsection{Low energy structure}

Up until recently it was believed that the underlying physics of SF ionisation were well understood with experimental results predominantly being interpreted in the context 


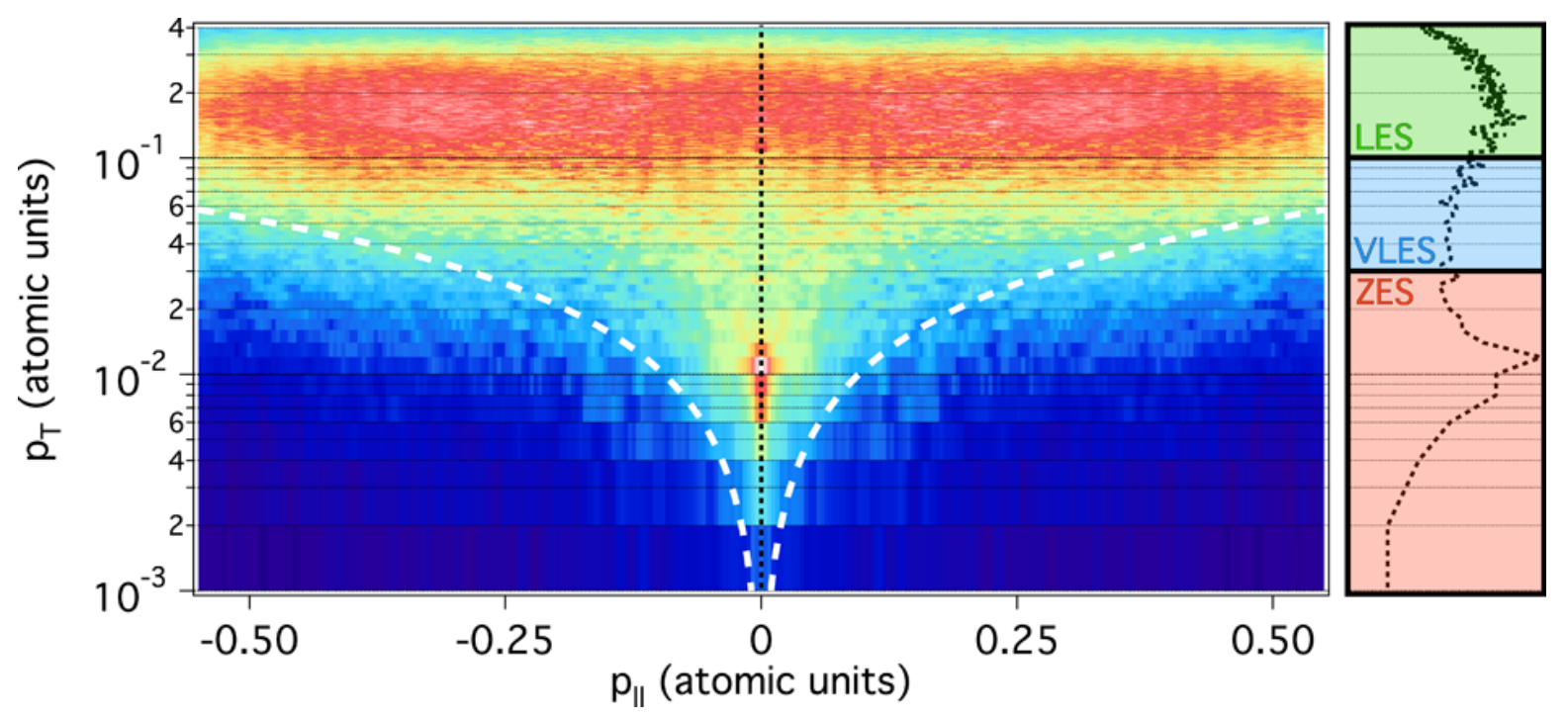

Figure 2. The complete low energy electron momentum map detected using a ReMi after the ionisation of randomly oriented $\mathrm{N}_{2}$ with the intense $3.1 \mu \mathrm{m}$ OPCPA based system. A cross-section of the momentum distribution taken at $p_{\|}=0$ is presented on the right side of the figure where the positions of the LES (green shading), VLES (blue shading) and ZES (red shading) regions in our experiment are also indicated. Typical TOF spectrometers only detect electrons below a $6^{\circ}$ acceptance angle (dashed white line).

of the popular strong-field approximation (SFA) [4, 8, 9]. With the recent progress in the development of intense mid-IR sources, probing deep into the QS regime has become possible. Unexpected low energy structure (LES) in the electron spectrum were observed using mid-IR wavelengths between 1.7 - $3.6 \mu \mathrm{m}$ in combination with a timeof-flight (TOF) spectrometer [10]. The LES was observed near $1 \mathrm{eV}$ and was found to be universal for all target species. It was found that the results presented in Ref. [10] were not reproducible by the SFA, which came as a surprise as it was expected to become more accurate as the QS limit was approached. The width of the LES increased with both laser wavelength and intensity, yet was found to be stable for constant $U_{P}$. The observation that it disappeared with circular polarisation hinted that its origin might be related to rescattering [11]. Initial calculations [12, 13], which attributed LES to an interplay of multiple forward scattering with a disturbance of the transverse electron momentum by the Coulomb field, indeed supported this proposition. Further investigations attributed the structure to bunching of electrons that miss the ionic core upon recollision (so-called 'soft recollisions') [14, 15]. In addition, improved SFA (ISFA) calculations incorporating a second-order rescattering term in the S-matrix expansion also show agreement with experimental results [16, 17].

Figure 2 presents a measured $\mathrm{N}_{2}$ electron distribution as a function of the initial electron longitudinal $\left(p_{\|}=p_{z}\right)$ and transverse $\left(p_{T}=\sqrt{p_{x}^{2}+p_{y}^{2}}\right)$ momenta after ionisation by the $3.1 \mu \mathrm{m}$ OPCPA source described below. A cross-section of the data taken at $p_{\|}=0$ (dotted vertical line) is presented on the right side of the figure and the 
transverse momentum range between 0.1 a.u. $<p_{T}<0.4$ a.u., corresponding to the LES region in our experiments, is shaded green. The peak near $p_{T}=0.15$ a.u. extends across the range -0.55 a.u. $<p_{\|}<0.55$ a.u. and corresponds to electron energies between $0.3 \mathrm{eV}<E<5 \mathrm{eV}$, which agrees well with the energy range in which the LES was observed in Refs. [10, 18]. Typical TOF spectrometers probe momentum distribution very close to the polarization axis so they only measure electrons below a $6^{\circ}$ acceptance angle cutoff (dashed white line).

\subsection{Very low energy structure}

Experimental investigations using similar wavelength lasers to Ref. [10] revealed socalled very low energy structures (VLESs) at even lower energies ( 100 meV) than the LES [18]. A ReMi detection system implemented by $\mathrm{Wu}$ et al. provided the ability to measure the initial momentum distributions of the electrons [19]. These results showed a distinctive double hump structure in the VLES longitudinal momentum distribution, which resembled structures observed previously using $0.8 \mu \mathrm{m}$ radiation [20]. The transverse momentum range in which we detect the VLES is shown as the shaded blue region roughly between 0.03 a.u. $<p_{T}<0.1$ a.u. in Fig. 2.

Theoretical understanding of the VLES is currently not as developed as for the LES. The structure has been attributed to an influence of the long-range Coulomb potential [19] or a Coulomb interaction at short distances [21], as well as being interpreted as second-order soft recollisions (i.e: second-order LES) [15, 22]. Recent SFA calculations, which included a second-order rescattering term, failed to reproduce the structure [16]. In addition, for experimental conditions similar to those in which the VLES was first observed, a suppression of the ionisation rate due to population trapping in Rydberg states has been reported [23]. Therefore, there seems to be some confusion as to what mechanisms contribute to the electrons detected in this region.

\subsection{Near zero energy structure}

More recently, we have probed even deeper into the QS regime using a high repetition rate $3.1 \mu \mathrm{m}$ OPCPA based source combined with a ReMi [24]. The ability to achieve Keldysh parameters approaching $\gamma \sim 0.1$ allowed the observation of a near zero energy structure $(\mathrm{ZES})$ at $\sim 1 \mathrm{meV}$. The ZES can clearly be observed in Fig. 2 within the shaded red region between 0.001 a.u. $<p_{T}<0.03$ a.u.. It is clear that the new structure is outside the detection range of typical TOF spectrometers, which might explain why it avoided observation until recently. The peak was located between 1$10 \mathrm{meV}$ for the studied species and is therefore well separated from the LES or VLES. The results showed that the ZES was as evident as the LES and even more visible than the VLES. Durá et al. [24] speculated that the origin of the ZES could be related to population trapping in Rydberg states or a rescattering mechanism.

So far no theoretical investigations have been published that reproduce the observed structure, so its exact origin still defies identification. Further high resolution 
measurements in the deep QS regime, which are currently underway, will provide insight into the problem.

\section{Experimental apparatus}

Previous experimental efforts aimed at investigating structure at low electron energies have predominantly used sources derived from the frequency conversion of Ti:Sapphire radiation at $0.8 \mu \mathrm{m}$ via difference frequency generation (DFG) [10] or parametric amplification $[10,18,19,23]$. A comparison of the laser sources used in the literature is presented in Fig. 3. Due to the unfavourable wavelength scaling of the ionisation rate at constant repetition rate $\left(\sim \lambda^{-1.2}\right.$ calculated using an intensity of $\left.1 \times 10^{14} \mathrm{~W} / \mathrm{cm}^{2}[25]\right)$, the detected yield decreases as one proceeds to probe further into the QS regime. The size of the data points in Fig. 3 scale logarithmically with the expected yield when both the wavelength scaling and repetition rate are accounted for. The ideal situation would be to be placed in the top right corner of this plot as this is where investigation deep in the QS regime can be achieved with the required high data acquisition rates. The dashed line at $5 \mathrm{kHz}$ represents an estimate of the repetition rate limit for the generation of intense mid-IR radiation using Ti:Sapphire pumped OPA and DFG in the near future.

The majority of the experiments in the literature have operated in the lower left corner of Fig. 3 using OPA based systems at wavelengths between $1.3-2.3 \mu \mathrm{m}$. These sources are typically intensity and repetition rate limited by the $0.8 \mu \mathrm{m}$ pump. A DFG based investigation with a wavelength of $3.6 \mu \mathrm{m}$ has been performed [10] but the same intensity and repetition rate limits apply. Durá et al. [24] recently presented the first results recorded using an OPCPA based system that operated at $3.1 \mu \mathrm{m}$ and was specifically designed for investigations appraoching the QS limit. OPCPA based technology offers many advantages over Ti:Sapphire based mid-IR generation using OPA or DFG. Particularly relevant to this study is the fact that intensities in the $10^{14} \mathrm{~W} / \mathrm{cm}^{2}$ range can be achieved at two orders of magnitude higher repetition rates. As the measured signal can be low when studying nonlinear phenomena, higher repetition rates become vital for keeping measurement times down. Another benefit of our particular OPCPA is that we are not intensity limited as we still have enough pump power available to implement further OPCPA stages if required.

The OPCPA based laser used in this work has been presented previously [5]. Briefly, it provides sub-six-cycle mid-IR pulses at a repetition rate of $160 \mathrm{kHz}$. The $3.1 \mu \mathrm{m}$ radiation is derived from DFG of $1.55 \mu \mathrm{m}$ and $1.05 \mu \mathrm{m}$ pulses in a magnesium oxide doped periodically poled lithium niobate (MgO:PPLN) crystal. It is subsequently chirped and parametrically amplified in four cascaded OPA stages before the pulse is compressed to $<60$ fs in a grating compressor. After focussing with a $50 \mathrm{~mm}$ parabolic mirror peak intensities above $10^{14} \mathrm{~W} / \mathrm{cm}^{2}$ can be achieved, corresponding to Keldysh parameters $\gamma<0.3$ for typical atomic and molecular targets. The $3.1 \mu \mathrm{m}$ radiation is the idler wave in all of the OPCPA stages, which in our case means that it is passively carrier envelope phase (CEP) stable [26]. Combining this capability with few-cycle 


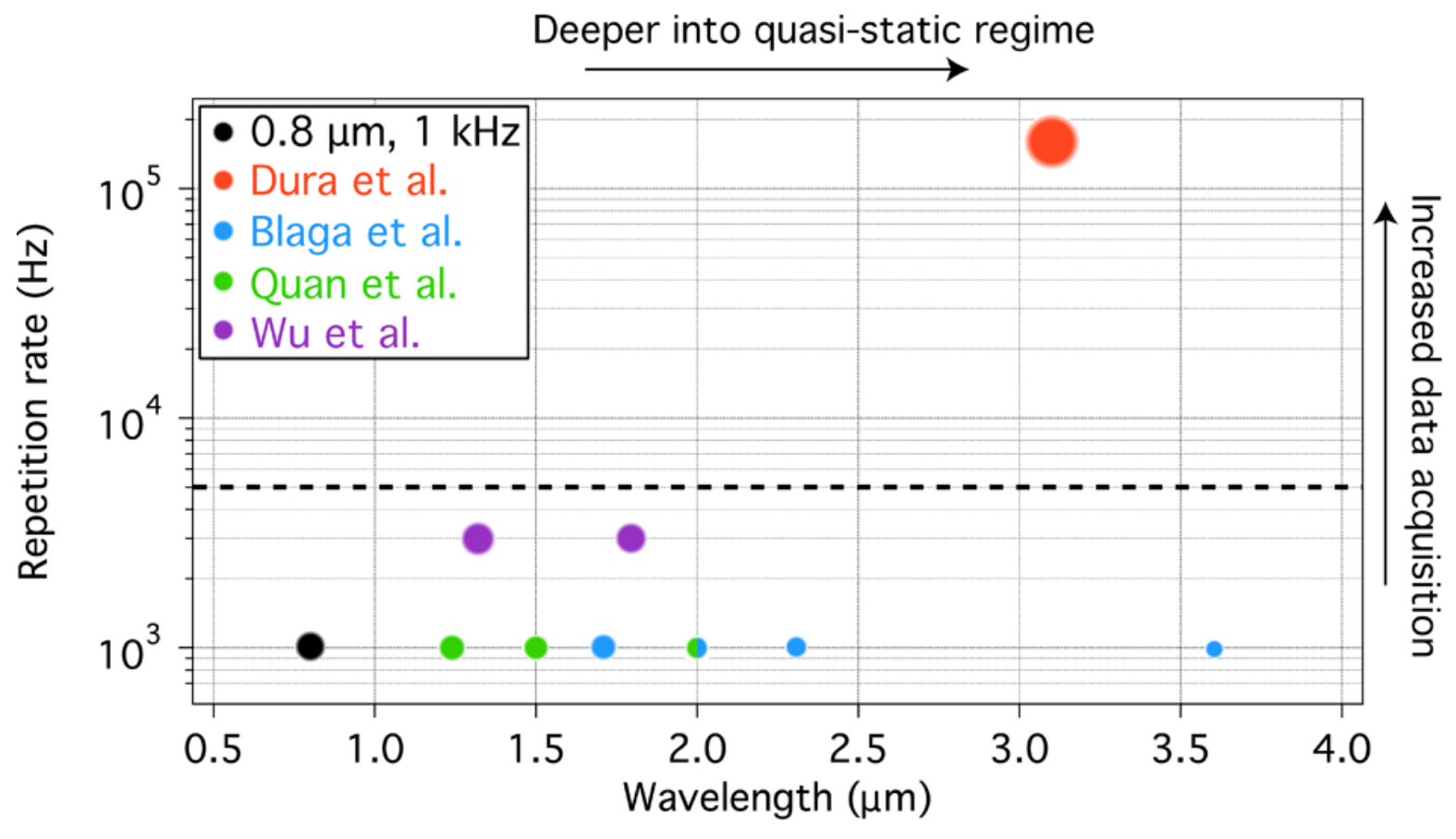

Figure 3. An overview of the laser systems that have been used to investigate low energy structures in the deep QS regime. The size of the data points scale logarithmically with the expected ionisation yield. The ideal laser would be located in the top-right of the image to ensure QS conditions at high data acquisition rates. Most experiments have been performed with Ti:Sapphire pumped OPA systems and are limited to the bottom left corner. Our $3.1 \mu \mathrm{m}$ OPCPA system operates at $160 \mathrm{kHz}$ and is therefore perfectly suited for the investigation of low energy structures. The dashed line at $5 \mathrm{kHz}$ represents an estimated future repetition rate limit for intense mid-IR radiation generated using Ti:Sapphire pumped OPA and DFG.

pulses, which we have already reported [27], opens the possibility of performing CEP resolved measurements of the LES, VLES and ZES in the near future.

In order to take advantage of the deep QS conditions generated by the mid-IR OPCPA we utilise a ReMi detection system. ReMis possess a number of benefits over typical TOF spectrometers such as the capability to detect electrons in coincidence with ions, directly extract the full three dimensional electron momentum distribution, and achieve electron energy resolutions at the meV level. For more details of the ReMi used here see the Methods section of Ref. [24] and for a thorough overview of the function and capabilities of ReMis in general see Ref. [28]. Briefly, we inject a cold supersonically expanded gas into vacuum via two skimming stages. The gas is ionised in the interaction region and the resultant charged particles are guided towards opposing position sensitive micro channel plate (MCP) detectors by homogenous electric and magnetic fields. Momentum distributions of both ions and electrons are then extracted from the position and time of detection. In the case of low electron energy detection the fields are low (an electric field of $E=1.5 \mathrm{~V} / \mathrm{cm}$ and a magnetic field of $B=4 \mathrm{G}$ ) to ensure high detection resolutions. A conservative estimate of upper bounds of the longitudinal and transerve 
momentum resolutions are $\Delta p_{\|}=0.011$ a.u. and $\Delta p_{T}=0.019$ a.u., respectively. The fields can also be increased to detect electrons with energies up to hundreds of $\mathrm{eV}$. The capability to resolve electron energies over a range of five orders of magnitude at high data acquisition rates showcases the versatility of this unique apparatus. It is perfectly suited to investigate the kinematics of low energy structures within the QS regime.

\section{Results and discussion}

We have measured high resolution electron momentum and energy distributions from Ar and isotropically oriented $\mathrm{N}_{2}$ in the deep QS regime. These targets complement each other with ionisation potentials of $15.76 \mathrm{eV}$ and $15.58 \mathrm{eV}$, respectively. Figure $4 \mathrm{a}$ presents the electron energy spectrum (linear binning) from Ar detected over four orders of magnitude between $1 \mathrm{meV}$ and $\sim 4 \mathrm{eV}$. The presented electron spectra have an upper energy limit for two reasons. Firstly, the ReMi is configured to provide high resolution imaging at low energy, which results in an transverse momentum detection limit of $p_{T}<0.4$ a.u.. Secondly, in post-processing we restrict our analysis to $\left|p_{\|}\right|<0.5$ a.u. to avoid false counts introduced by nodal structures from the magnetic field induced electron cyclotron motion.

The black curve in Fig. 4a represents the electron energy spectrum measured for $\mathrm{Ar}$ when all electrons within the LES, VLES and ZES momentum regions are analysed. A broad peak between $0.2-3 \mathrm{eV}$ can be observed that corresponds to the LES presented in Ref. [10]. In addition, a narrow peak between $3-10$ meV can clearly be discerned. This corresponds to the ZES first presented in Ref. [24]. The VLES peak, which has previously been observed near $100 \mathrm{meV}[18,19]$, cannot directly be observed in the total energy spectrum. However, if the electron selection is limited to the VLES momentum range between 0.02 a.u. $<p_{T}<0.1$ a.u., as indicated in Fig. 2, then it becomes more evident (blue curve). The VLES contributions are masked by the LES electrons at higher energies and the ZES electrons at lower energies. Analysis has confirmed that if an acceptance angle limit is enforced during post processing, such as in Wu et al., then the VLES peak becomes visible in the electron energy spectrum. The different contributions to the overall spectra for the LES (green curve) and the ZES (red curve) momentum ranges are also presented in Fig. 4a.

Further information can be gained by presenting the parallel momentum distributions of the LES, VLES and ZES regions integrated over their respective $p_{T}$ limits (as indicated in Fig. 2). The results of this procedure are presented for Ar in Fig. $4 \mathrm{~b}$ where each curve has been rescaled between zero and one to highlight the observed features. For reference the result of integrating over the entire $p_{T}$ range (all electrons) is also shown. The most striking feature is the ZES peak at $p_{\|}=0$ a.u. that has a full width at half maximum (FWHM) width of 0.23 a.u. $(\sim 0.7 \mathrm{eV})$. The structure observed in the momentum range attributed to the VLES region drastically contrasts this peak in two ways. Firstly, the peak has split down the middle with a depth that decreases to about 0.8 . This feature shows some resemblance to the double 

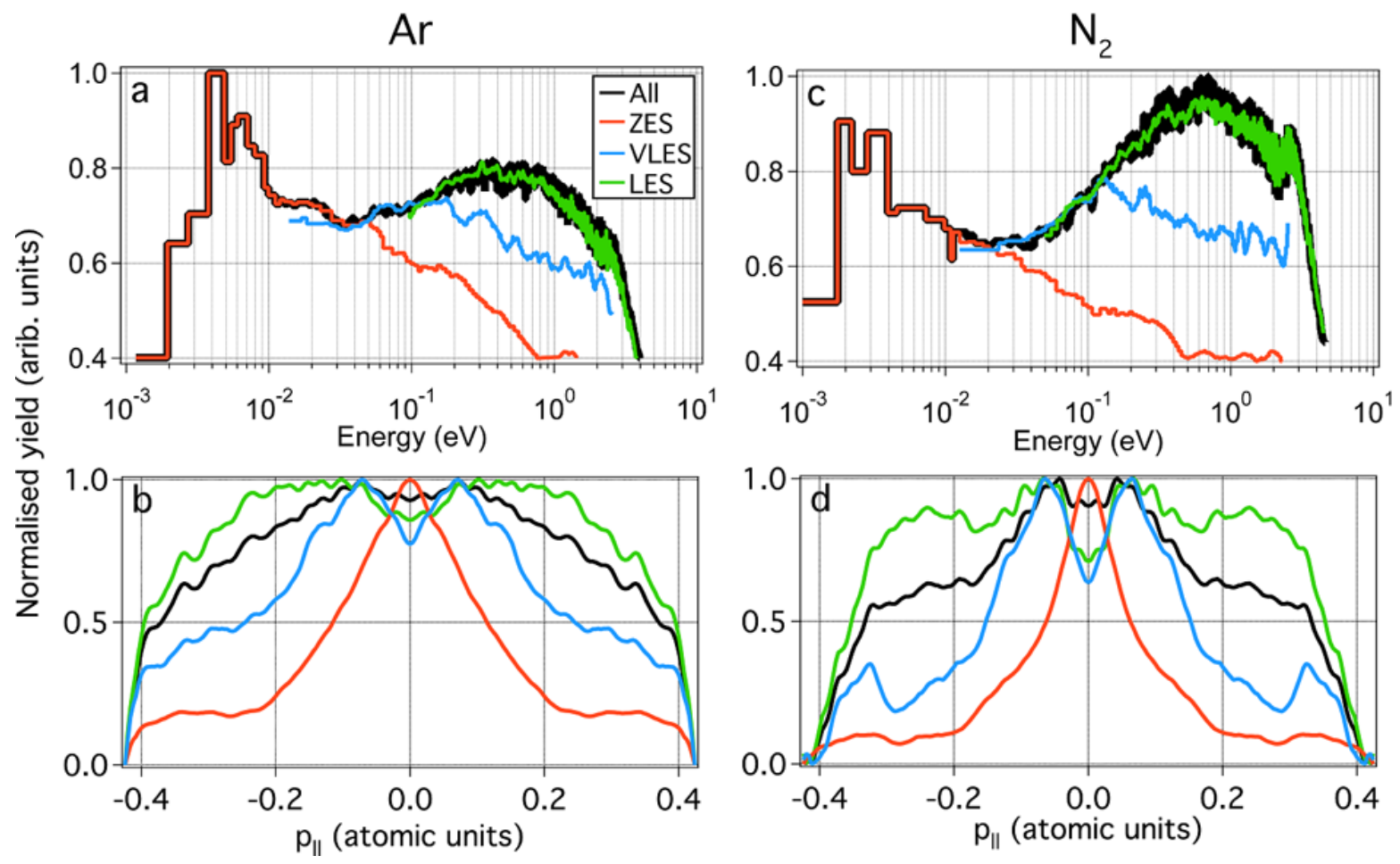

Figure 4. The detected electron energy spectra for $\mathrm{Ar}$ (a) and $\mathrm{N}_{2}$ (b) with the different low energy regions indicated by colour. Also shown is the obtained spectra when electrons from all of the low energy regions are included. The corresponding longitudinal momentum distributions are also shown for $\mathrm{Ar}$ (b) and $\mathrm{N}_{2}$ (d).

peak structure previously reported in Ref. [19]. Secondly, the FWHM has increased to 0.48 a.u. $(3.1 \mathrm{eV})$. As the $p_{T}$ integration range continues to be increased to values corresponding to the LES region the double hump structure starts to smear out [19]. The FWHM of the LES region has increased to 0.79 a.u. $(8.5 \mathrm{eV})$. When all electrons are included in the distribution, the ZES single peak signal is completely washed out and the VLES double hump structure is significantly suppressed.

The results for the ionisation of $\mathrm{N}_{2}$ are presented in Figs. 4c \& 4d. The ZES peak in the energy spectrum is at a slightly lower energy of $2 \mathrm{meV}$ while the LES peak is still located near $1 \mathrm{eV}$. The contributions from the VLES electrons are again obscured in the overall spectrum but the peak near $100 \mathrm{meV}$ becomes more obvious once they are isolated in momentum space. The other noticeable difference between the Ar and $\mathrm{N}_{2}$ energy spectra is that the LES is comparatively more prominent than the ZES in the case of $\mathrm{N}_{2}$. The longitudinal momentum distributions also reveal some other differences between the two targets. Firstly, the widths of the ZES and VLES regions have decreased to FWHMs of 0.12 a.u. $(0.2 \mathrm{eV})$ and 0.30 a.u. $(1.2 \mathrm{eV})$, respectively. Secondly, the VLES double peak depth now reaches to 0.64. Finally, the VLES distribution possesses well defined 'shoulders' that are either absent or much less obvious for Ar.

Theoretical investigations into the origins of the LES $[12,13,14,15,22]$ and the VLES [19] have uncovered a wealth of information. However, no published simulations 

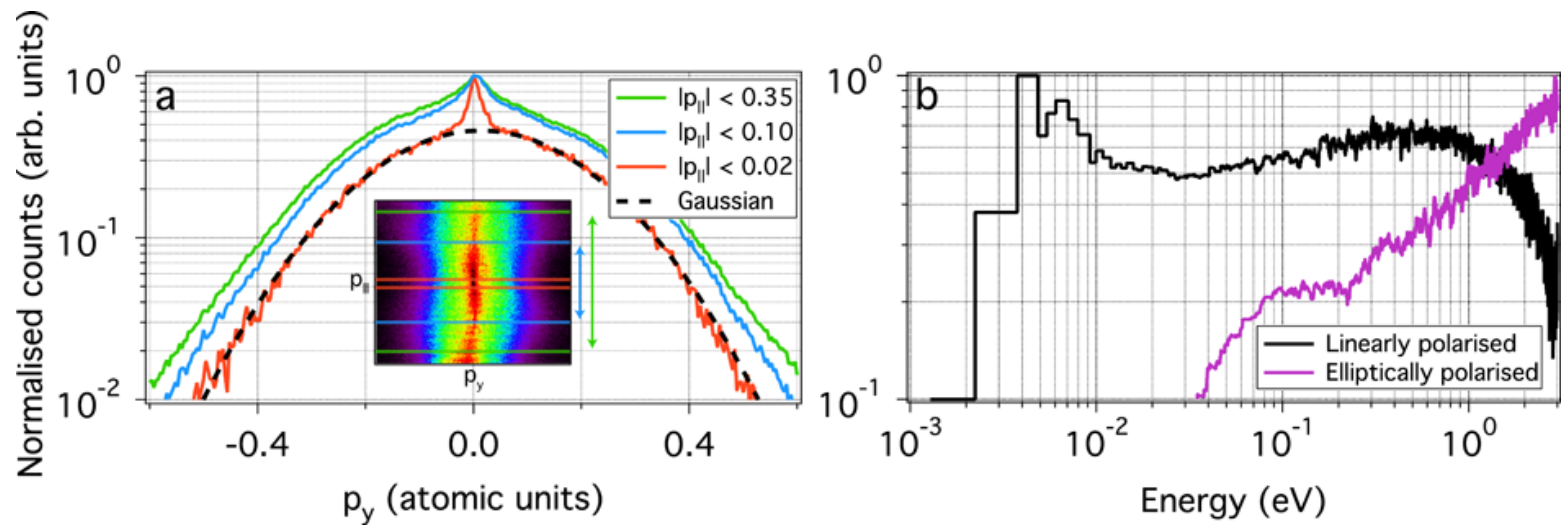

Figure 5. $\mathrm{N}_{2}$ momentum distributions in one perpendicular direction for different parallel momentum ranges are presented in (a). The ranges roughly correspond to the $p_{\|}$values at which the LES, VLES and ZES regions are observed to start and are graphically indicated in the inset. A Gaussian fit to the ZES curve is presented to highlight the deviation of the cusp-like feature at $p_{y}=0$. The detected Ar electron energy spectra for linearly and elliptically polarised light is presented in (b). The ZES structure completely disappears for elliptical polarisation yet some LES and VLES signal persists.

have been able to successfully reproduce the ZES since it was first reported [24]. We are therefore in the unusual position where experimental progress seems to be ahead of current SF ionisation understanding. This situation is reminiscent of the initial surprising LES results that Blaga et al. reported. To help decipher the ZES mystery, we performed additional experiments which may provide some valuable insight into the low energy kinematics that are at play.

Investigation performed by Rudenko et al. [29] pointed out that cusp-like structures in one of the perpendicular momentum distributions are unambiguous signatures of long range interactions with the Coulomb field. Figure 5a presents the observed normalised $p_{y}$ distributions for $\mathrm{N}_{2}$ over three different $p_{\|}$integration ranges that roughly correspond to the $p_{\|}$values at which the LES, VLES and ZES regions are observed to start (green, blue and red curves, respectively). The cusp-like structure can clearly be identified in each region but it is particularly obvious for the ZES region. A Gaussian fit to the ZES data, without including the cusp-like structure, is also presented as the dashed black curve. The fit is excellent for the range $\left|p_{y}\right|>0.04$. These results seem to be universal to all tested targets and they indicate that the Coulomb field is indeed playing a role in the creation of the ZES.

Figure 5b presents the energy spectrum of Ar for both linearly and $15 \%$ elliptically polarised light. Both curves have been normalised to emphasise their features. The linearly polarised data is similar to that presented in Fig. 4a and shows the familiar ZES and LES peaks. Clearly, the ZES is absent for the elliptically polarised case yet electrons in the VLES and LES regions can still be observed. In fact, a small VLES plateau near $100 \mathrm{meV}$ is visible. If the LES and VLES are interpreted as being due to soft-recollisions $[15,22]$ then the fact that the ZES is absent might point to another 
mechanism being responsible. It is possible that the ZES is due to population trapping in high lying Rydberg states as it has previously been shown that elliptically polarised light reduces these populations [30]. Further work into the origin is ongoing and will be published at a further date.

\section{Conclusion}

We have presented full three dimensional kinematic measurements of the ionisation of $\mathrm{Ar}$ and $\mathrm{N}_{2}$ deep in the QS regime. We can resolve the LES, VLES and the newly discovered ZES regions simultaneously in both energy and momentum space. The unique combination of a high repetition rate mid-IR OPCPA laser with a reaction microscope was specifically designed to investigate these low energy structures at high resolution.

We have found that the ZES region seems to be present for all of the atoms and molecules investigated so far ( $\mathrm{Ar}$ and $\mathrm{N}_{2}$ here and also $\mathrm{O}_{2}$ in Durá et al. [24]). Its origin is still unknown but initial evidence points towards population trapping in high lying states. Further measurements at the high resolutions offered by our system will complement theoretical efforts to track down the responsible mechanism.

\section{Acknowledgements}

We acknowledge support from MINISTERIO DE ECONOMA Y COMPETITIVIDAD through Plan Nacional (FIS2011-30465-C02-01), the Catalan Agencia de Gestio dAjuts Universitaris i de Recerca (AGAUR) with SGR 2009-2013, Fundacio Cellex Barcelona, and funding from LASERLAB-EUROPE, grant agreement 228334.

\section{References}

[1] J. Itatani, J. Levesque, D. Zeidler, H. Niikura, H. Pépin, J. C. Kieffer, P. B. Corkum, and D. M. Villeneuve. Tomographic imaging of molecular orbitals. Nature, 432:867, 2004.

[2] C. I. Blaga, J. Xu, A. D. DiChiara, E. Sistrunk, Zhang K., P. Agostini, T. A. Miller, L. F. DiMauro, and C. D. Lin. Imaging ultrafast molecular dynamics with laser-induced electron diffraction. Nature, 483:194, 2012.

[3] F. Krausz and M. Ivanov. Attosecond physics. Rev. Mod. Phys., 81:163, 2009.

[4] L. V. Keldysh. Ionization in the Field of a Strong Electromagnetic Wave. Sov. Phys. JETP, 20:1307, 1965.

[5] A. Thai, M. Hemmer, P. K. Bates, O. Chalus, and J. Biegert. Sub-250-mrad, passively carrier-envelope-phase-stable mid-infrared OPCPA source at high repetition rate. Opt. Lett., 36(19):3918, 2011.

[6] A. M. Perelemov, V. S. Popov, and M. V. Terent'ev. Ionization of atoms in an alternating electric field. Sov. Phys. JETP, 23:924, 1966.

[7] T. Popmintchev, M.-C. Chen, D. Popmintchev, P. Arpin, S. Brown, S. Ališauskas, G. Andriukaitis, T. Balciunas, O. D. Mücke, A. Pugzlys, A. Baltuška, B. Shim, S. E. Schrauth, A. Gaeta, C. Hernández-García, L. Plaja, A. Becker, A. Jaron-Becker, M. M. Murnane, and H. C. Kapteyn. Bright coherent ultrahigh harmonics in the kev x-ray regime from mid-infrared femtosecond lasers. Science, 336(6086):1287, 2012. 
[8] F. H. M. Faisal. Multiple absorption of laser photons by atoms. J. Phys. B: At. Mol. Opt. Phys., 6:L89, 1973.

[9] H. R. Reiss. Effect of an intense electromagnetic field on a weakly bound system. Phys. Rev. A, 22:1786, 1980.

[10] C. I. Blaga, F. Catoire, P. Colosimo, G. G. Paulus, H. G. Muller, P. Agostini, and L. F. DiMauro. Strong-field photoionization revisited. Nat. Phys., 5:335, 2009.

[11] F. H. M. Faisal. Strong-field physics: Ionization surprise. Nat. Phys., 5:319, 2009.

[12] C. Liu and K. Z. Hatsagortsyan. Origin of unexpected low energy structure in photoelectron spectra induced by midinfrared strong laser fields. Phys. Rev. Lett., 105:113003, 2010.

[13] T.-M. Yan, S. V. Popruzhenko, M. J. J. Vrakking, and D. Bauer. Low-energy structures in strong field ionization revealed by quantum orbits. Phys. Rev. Lett., 105:253002, 2010.

[14] C. Lemell, K. I. Dimitriou, X.-M. Tong, S. Nagele, D. V. Kartashov, J Burgdörfer, and S. Gräfe. Low-energy peak structure in strong-field ionization by midinfrared laser pulses: Two-dimensional focusing by the atomic potential. Phys. Rev. A, 85:011403(R), 2012.

[15] A. Kästner, U. Saalmann, and J. M. Rost. Electron-energy bunching in laser-driven soft recollisions. Phys. Rev. Lett., 108:033201, 2012.

[16] L. Guo, S. S. Han, X. Liu, Y. Cheng, Z. Z. Xu, J. Fan, J. Chen, S. G. Chen, W. Becker, C. I. Blaga, A. D. DiChiara, E. Sistrunk, P. Agostini, and L. F. DiMauro. Scaling of the Low-Energy Structure in Above-Threshold Ionization in the Tunneling Regime: Theory and Experiment. Phys. Rev. Lett., 110:013001, 2013.

[17] D. B. Milošević. Reexamination of the improved strong-field approximation: Low-energy structures in the above-threshold-ionization spectra for short-range potentials. Phys. Rev. A, 88:023417, 2013.

[18] W. Quan, Z. Lin, M. Wu, H. Kang, H. Liu, X. Liu, J. Chen, J. Liu, X. T. He, S. G. Chen, H. Xiong, L. Guo, H. Xu, Y. Fu, Y. Cheng, and Z. Z. Xu. Classical Aspects in Above-Threshold Ionization with a Midinfrared Strong Laser Field. Phys. Rev. Lett., 103:093001, 2009.

[19] C. Y. Wu, Y. D. Yang, Y. Q. Liu, Q. H. Gong, M. Wu, X. Liu, X. L. Hao, W. D. Li, X. T. He, and J. Chen. Characteristic Spectrum of Very Low-Energy Photoelectron from Above-Threshold Ionization in the Tunneling Regime. Phys. Rev. Lett., 109:043001, 2012.

[20] A. Rudenko, K. Zrost, C. D. Schröter, V. L. B. de Jesus, B. Feuerstein, R. Moshammer, and J. Ullrich. Resonant structures in the low-energy electron continuum for single ionization of atoms in the tunnelling regime. J. Phys. B: At. Mol. Opt. Phys., 37:L407, 2004.

[21] L. Zhi-Yang, W. Ming-Yan, Q. Wei, L. Xiao-Jun, C. Jing, and C. Ya. Coulomb singularity in the transverse momentum distribution for strong-field single ionization. Chin. Phys. B, 23:02201, 2014.

[22] C. Lemell, J Burgdörfer, S. Gräfe, K. I. Dimitriou, D. G. Arbó, and X.-M. Tong. Classical-quantum correspondence in atomic ionization by midinfrared pulses: Multiple peak and interference structures. Phys. Rev. A, 87:013421, 2013.

[23] H. Liu, Y. Liu, L. Fu, G. Xin, D. Ye, J. Liu, X. T. He, Y. Yang, X. Liu, Y. Deng, C. Wu, and Q. Gong. Low yield of near-zero-momentum electrons and partial atomic stabilization in strong-field tunneling ionization. Phys. Rev. Lett., 109:093001, 2012.

[24] J. Dura, N. Camus, A. Thai, A. Britz, M. Hemmer, M. Baudisch, A. Senftleben, C. D. Schroöter, J. Ullrich, R. Moshammer, and J. Biegert. Ionization with low-frequency fields in the tunneling regime. Sci. Rep., 3:2675, 2013.

[25] S. V. Popruzhenko, V. D. Mur, V. S. Popov, and D. Bauer. Strong field ionization rate for arbitrary laser frequencies. Phys. Rev. Lett., 101:193003, 2008.

[26] A. Baltuška, T. Fuji, and T. Kobayashi. Controlling the carrier-envelope phase of ultrashort light pulses with optical parametric amplifiers. Phys. Rev. Lett., 88:133901, 2002.

[27] M. Hemmer, M. Baudisch, A. Thai, A. Couairon, and J. Biegert. Self-compression to sub-3-cycle duration of mid-infrared optical pulses in dielectrics. Opt. Exp., 21:28095, 2013.

[28] J. Ullrich, R. Moshammer, A. Dorn, R. Dörner, L. P. H. Schmidt, and H. Schmidt-Böcking. Recoil- 
ion and electron momentum spectroscopy: reaction-microscopes. Rep. Prog. Phys., 66:1463, 2003.

[29] A. Rudenko, K. Zrost, T. Ergler, A. B. Voitkiv, B. Najjari, V. L. B. de Jesus, B. Feuerstein, C. D. Schröter, R. Moshammer, and J. Ullrich. Coulomb singularity in the transverse momentum distribution for strong-field single ionization. J. Phys. B: At. Mol. Opt. Phys., 38:L191, 2005.

[30] T. Nubbemeyer, K. Gorling, A. Saenz, U. Eichmann, and W. Sandner. Strong-field tunneling without ionization. Phys. Rev. Lett., 101:233001, 2008. 